\title{
Size Effect in Heterogeneous Nucleation
}

Cite as: J. Chem. Phys. 29, 572 (1958); https://doi.org/10.1063/1.1744540

Submitted: 16 May 1958 . Published Online: 13 August 2004

N. H. Fletcher

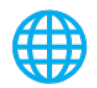

View Online

\section{ARTICLES YOU MAY BE INTERESTED IN}

Heterogeneous nucleation or homogeneous nucleation?

The Journal of Chemical Physics 112, 9949 (2000); https://doi.org/10.1063/1.481644

Spatial control in the heterogeneous nucleation of water

Applied Physics Letters 95, 094101 (2009); https://doi.org/10.1063/1.3200951

Rate of Nucleation in Condensed Systems

The Journal of Chemical Physics 17, 71 (1949); https://doi.org/10.1063/1.1747055

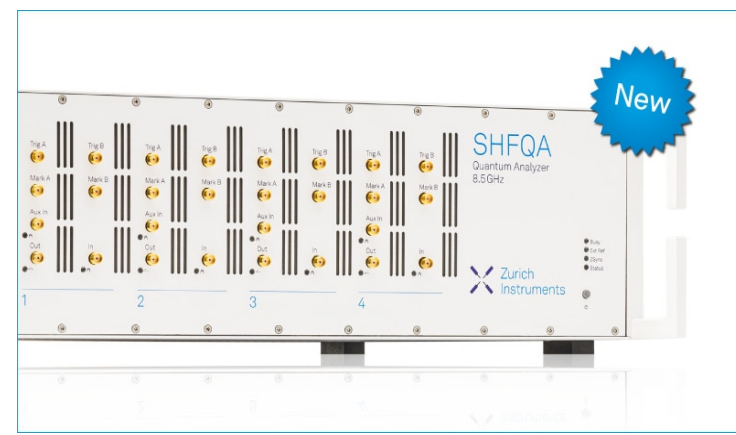

\section{Your Qubits. Measured.}

Meet the next generation of quantum analyzers

- Readout for up to 64 qubits

- Operation at up to $8.5 \mathrm{GHz}$, mixer-calibration-free

- Signal optimization with minimal latency

\section{Find out more}

Zurich

$\checkmark$ Instruments 
74, 4656 (1952); for $n-\mathrm{C}_{5} \mathrm{H}_{11} \mathrm{SH}$ : H. L. Finke et al., J. Am. Chem. Soc. 74, 2804 (1952); $t$ - $\mathrm{C}_{4} \mathrm{H}_{9} \mathrm{SH}: \mathrm{J}$. P. McCullough et al., J. Am. Chem. Soc. 75, 1818 (1953); $i$ - $\mathrm{C}_{3} \mathrm{H}_{7} \mathrm{SH}$ : J. P. McCullough et al., J. Am. Chem. Soc. 76, 4796 (1954); $i-\mathrm{C}_{3} \mathrm{H}_{7} \mathrm{SCH}_{3}$ : J. P. McCullough et al., J. Am. Chem. Soc. 77, 6119 (1955). For $n-\mathrm{C}_{3} \mathrm{H}_{7} \mathrm{SH}$ : R. E. Pennington et al., J. Am. Chem. Soc. 78, 3266 (1956).

(3) Values for $n-\mathrm{C}_{4} \mathrm{H}_{9} \mathrm{SH}$ and $n-\mathrm{Pr}-\mathrm{S}-\mathrm{CH}_{3}$ : D. W. Scott et al., J. Am. Chem. Soc. 79, 1062 (1957).

\section{F. Miscellaneous}

(1) $C_{p}$ and $S^{\circ}$ for $\mathrm{SiF}_{4}$ : Davis, Cleveland, and Meister, J. Chem. Phys. 20, 454 (1952). See also J. Chem. Phys. 20, 1498 (1952).

(2) $C_{p}$ and $S^{\circ}$ for $\mathrm{SiH}_{3} \mathrm{I}:$ R. N. Dixon and N. Shep- pard, Trans. Faraday Soc. 53, 282 (1957); for $\mathrm{SiF}_{3} \mathrm{CH}_{3}$ : R. L. Collins and J. R. Nielsen, J. Chem. Phys. 23, 329 (1955) ; for $\mathrm{SiH}_{4}$ : A. P. Altschuler, J. Chem. Phys. 23, 761 (1955).

(3) $C_{p}$ and $S^{\circ}$ for $\mathrm{SiH}_{3} \mathrm{Br}$ and $\mathrm{SiH}_{2} \mathrm{Br}_{2}$ : Mayo, Opitz, and Peake, J. Chem. Phys. 23, 1344 (1955). $C_{p}$ and $S^{\circ}$ for the mixed $\mathrm{Br}$ and $\mathrm{Cl}$ silanes: B. Schneider and J. Pliva, Chem. listy 48, 336 (1954) and C. Cerny and E. Erdos, Chem. listy 47, 1742 (1953).

(4) $\mathrm{S}^{\circ}$ for $\mathrm{Si}\left(\mathrm{CH}_{3}\right)_{4}:$ J. G. Aston, J. Am. Chem. Soc. 63, 2343 (1941).

(5) $C_{p}$ and $S^{\circ}$ for $\mathrm{GeH}_{4}, \mathrm{GeH}_{3} \mathrm{Cl}$, and $\mathrm{GeD}_{3} \mathrm{Cl} ; \mathrm{R}$. G. Lord and C. M. Steise, J. Chem. Phys. 22, 542 (1954). (6) Values for $\mathrm{P}_{2}, \mathrm{P}_{4}, \mathrm{PH}_{3}, \mathrm{PCl}_{3}, \mathrm{PCl}_{5}, \mathrm{POCl}_{3}, \mathrm{PBr}_{3}$, $\mathrm{PN}, \mathrm{AsF}_{3}, \mathrm{AsCl}_{3}, \mathrm{SbCl}_{3}, \mathrm{BiCl}_{3}$, and $\mathrm{SiCl}_{4}$ form NBS Circular No. 500.

\title{
Size Effect in Heterogeneous Nucleation
}

\author{
N. H. FLETCHER \\ C.S.I.R.O., Radiophysics Laboratory, Sydney, Australia \\ (Received May 16, 1958)
}

\begin{abstract}
Assuming a spherical nucleating particle, the effect of particle size and surface properties upon nucleation efficiency is investigated. A general result is derived which is then applied to the condensation, sublimation, and freezing of water on foreign nuclei. The size effect is found to become important in the range 100-1000 A of particle radius. For particles larger than this, nucleation efficiency is substantially independent of size, while for smaller particles the efficiency is very greatly reduced.
\end{abstract}

\section{INTRODUCTION}

$\mathbf{T}$ HE heterogeneous nucleation of supercooled or supersaturated solutions by foreign particles is of interest in many fields, and an understanding of the dependence of nucleation efficiency on the size and surface properties of the nucleating particles is of great importance. The present study is related particularly to processes of condensation, sublimation, and freezing of water in the presence of natural or artificial aerosol particles, where the results have immediate application to problems in cloud physics. The theory is, however, more general than this and has, as far as possible, been stated in a form which can easily be applied to other situations.

The general theory of nucleation processes of this kind is now well established and we shall not need to re-examine its fundamentals. ${ }^{1}$

The rate of formation of critical embryos which can then develop into macroscopic droplets or crystals is given by an expression of the form

$$
J=K \exp \left(-\Delta G^{*} / k T\right),
$$

${ }^{1} \mathrm{~A}$ recent survey has been given by $W . J$. Dunning in Chemistry of the Solid State, edited by $\mathrm{W}$. E. Garner (Butterworths Publications, Ltd., London, 1955) p. 159. where $K$ is a kinetic constant, and $\Delta G^{*}$ is the free energy of formation of a critical embryo on the nucleating particle. The value of $K$ is somewhat uncertain and depends in detail upon the nucleation situation, but fortunately it is sufficient to know its value to one or two orders of magnitude. $J$ is determined largely by $\Delta G^{*}$, and most of our discussion will be devoted to determination of this quantity.

\section{CRITICAL FREE ENERGY}

We shall assume that the nucleating particle is a sphere. This is necessary for mathematical simplicity, but it also physically reasonable, since in the size range where shape effects enter, particles made by many processes tend to be spherical. In the interests of simplicity, we are forced to neglect the differences between various crystal faces and use instead average values for the quantities involved. This is unlikely to have more than a small effect in most cases.

Similar assumptions are made about the embryo, this being a portion of a sphere as shown in Fig. 1 . It may be objected that this is an oversimplification for crystalline embryos, but in the situations we envisage the crystal is very small, less than 100 mole- 
cules across, and since it is near its melting point its surface is probably "rough" in the sense of Burton and Cabrera. ${ }^{2}$ These two considerations make it likely that the embryo will be spherical to a reasonable approximation.

The application of macroscopic concepts like surface free energy to very small groups of molecules has received considerable attention in the literature, with the general conclusion that these concepts are applicable and the deviation from their macroscopic values is small, even for embryos containing as few as 100 molecules. One new quantity becomes appreciable, however, for small embryos. This is the free energy associated with edges. This had been discussed to some extent by Frenkel $^{3}$ and by Wylie, ${ }^{4}$ but we shall have to neglect its effects in the present discussion since no physical data are available.

Referring to Fig. 1, we denote the parent phase by subscript 1 , the embryo by 2 , and the nucleus by 3 . If we denote volume by $V$ and surface area by $S$, then the free energy of formation of an embryo of radius $r$ on a nucleus of radius $R$ is

$$
\Delta G=\Delta G_{v} V_{2}+\sigma_{12} S_{12}+\left(\sigma_{23}-\sigma_{13}\right) S_{23},
$$

where $\Delta G_{v}$ is the free energy difference per unit volume of phase 2 between matter in state 1 and matter in state 2 , and $\sigma_{i j}$ is the surface free energy of the interface between phases $i$ and $j$. If we write,

$$
m=\cos \theta=\left(\sigma_{13}-\sigma_{23}\right) / \sigma_{12},
$$

then this is the usual definition of the contact angle $\theta$, provided $-1 \leq m \leq 1$.

Referring again to Fig. 1 we see that,

$$
\left.\begin{array}{l}
S_{12}=2 \pi r^{2}(1-\cos \psi), \\
S_{23}=2 \pi R^{2}(1-\cos \varphi), \\
V_{2}=\frac{1}{3} \pi r^{3}\left(2-3 \cos \psi+\cos ^{3} \psi\right) \\
\quad-\frac{1}{3} \pi R^{3}\left(2-3 \cos \varphi+\cos ^{3} \varphi\right)
\end{array}\right\}
$$

and

$$
\left.\begin{array}{l}
\cos \varphi=(R-r \cos \theta) / d=(R-r m) / d, \\
\cos \psi=-(r-R \cos \theta) / d=-(r-R m) / d,
\end{array}\right\}
$$

where

$$
d=\left(R^{2}+r^{2}-2 r R m\right)^{\frac{1}{2}} .
$$

To evaluate the critical free energy $\Delta G^{*}$ we can substitute the expressions (4) into (2) and require that

$$
(\partial G / \partial r)^{*}=0
$$

${ }^{2}$ W. K. Burton and N. Cabrera, Discussions Faraday Soc., No. $5,33(1949)$

${ }^{3}$ J. Frenkel, Kinetic Theory of Liquids (Oxford University Press, New York, 1946), p. 403.

${ }^{4}$ R. G. Wylie, 1956 (unpublished).

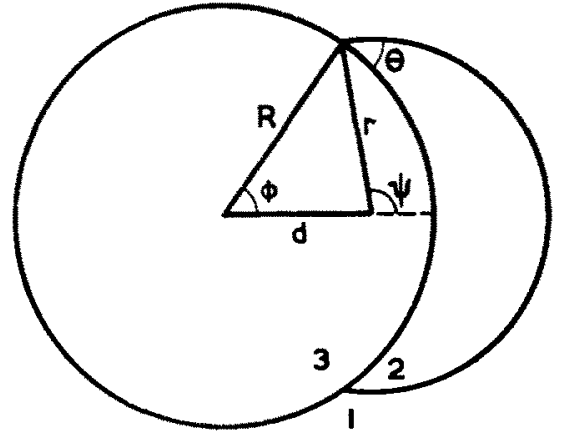

Fic. 1. Embryo 2 on nucleating particle 3 in parent phase 1.

This involves a great amount of labor, however, and instead we note that, independently of the shape or even presence of the nucleus, the critical radius $r^{*}$ must satisfy the usual nucleation equation

$$
r^{*}=-2 \sigma_{12} / \Delta G_{v} .
$$

This is necessarily true since all parts of the embryo surface must be in equilibrium with the metastable phase 1 . It can be verified by substitution in (7).

Now substituting the expressions (3)-(8) into Eq. (2) and writing

$$
x=R / r^{*},
$$

the free energy of formation of a critical embryo is

$$
\Delta G^{*}=\frac{8 \pi \sigma_{12}{ }^{3}}{3\left(\Delta G_{v}\right)^{2}} f(m, x)
$$

where

$$
\begin{aligned}
f(m, x)=1+\left(\frac{1-m x}{g}\right)^{3}+x^{3}\left[2-3\left(\frac{x-m}{g}\right)+\left(\frac{x-m}{g}\right)^{3}\right] \\
+3 m x^{2}\left(\frac{x-m}{g}-1\right),
\end{aligned}
$$

and

$$
g=\left(1+x^{2}-2 m x\right)^{\frac{1}{2}}
$$

This expression agrees exactly with that for homogeneous nucleation when $x=0$ and with that given by Turnbull and Vonnegut ${ }^{5}$ for nucleation on a plane surface when $x=\infty$. It disagrees, however, with the expression derived for the same situation by Dufour and Defay ${ }^{6}$ and for the simpler case $m=0$ by these authors and by Krastanow, ${ }^{7}$ though these all agree with our result in the limits $x=0$ and $\infty$.

The errors in these earlier treatments are sufficiently important to be pointed out. In the first place, while the free energy of formation of a free spherical embryo of critical size is $\frac{1}{3} \sigma S$, this is only true for embryos of

\footnotetext{
${ }^{5}$ D. Turnbull and B. Vonnegut, Ind. Eng. Chem. 44, 1292 (1952).

${ }^{6}$ L. Dufour and R. Defay, Tellus 5, 293 (1953).

${ }^{7}$ L. Krastanow, Meteorol. Z. 57, No. 10, 357 (1940); 58, No. $2,37(1941)$
} 
critical size and not for an arbitrary radius. Secondly, an expression of the form $\frac{1}{3} \alpha \sigma S$ where $\alpha$ is a constant, only applies for critical embryos of constant external shape, for which

$$
\begin{aligned}
& V_{2}=A r^{3}, \\
& S_{2}=B r^{3},
\end{aligned}
$$

and these conditions are not satisfied for a spherical nucleating particle. There is an additional error in the treatment of Dufour and Defay, which is in the nature of an invalid approximation [their Eq. (16)], which we shall not consider further here.

In Fig. 2 is shown the expression $f(m, x)$ as a function of $x$ for various values of the parameter $m$. Using an appropriate value from this graph and inserting values for $\sigma_{12}$ and $\Delta G_{v}$, Eq. (10) can be used to calculate $\Delta G^{*}$ for any nucleation process.

\section{KINETIC COEFFICIENT}

As was remarked in the introduction, there is some uncertainty about the value of the kinetic coefficient $K$ in Eq. (1), due in part to uncertainties in the mechanism of addition of molecules to the growing embryo. Some of these calculations have been discussed by Dunning ${ }^{1}$ without reaching any clear conclusion, and the coefficient is obviously different for different situations. For our calculation we shall adopt the value $10^{25} \mathrm{~cm}^{-2} \mathrm{sec}^{-1}$. This is probably correct within a few orders of magnitude for all the processes we shall consider, and the final result is in any case extremely insensitive to this coefficient. For processes where the kinetic coefficient is found to differ by more than a few orders of magnitude, an appropriate correction to our final result (14) is easily made.

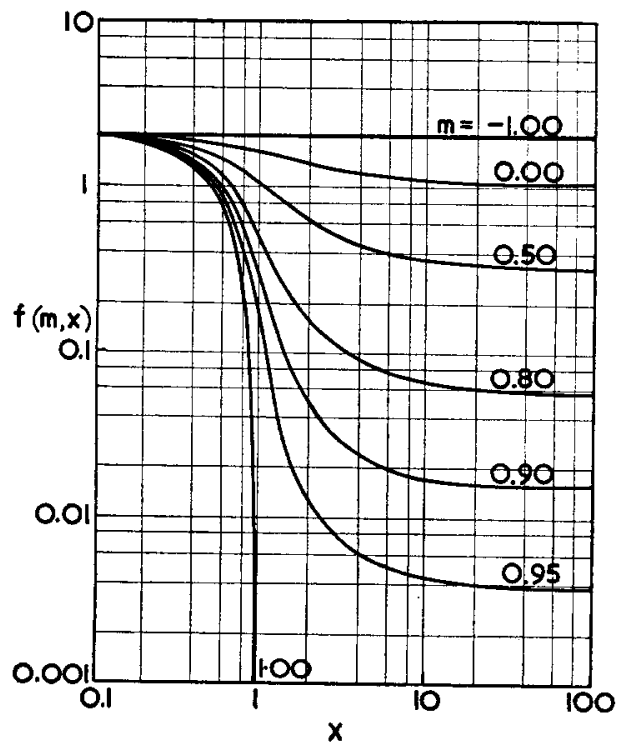

FrG. 2. The geometrical factor $f(m, x)$ in terms of the ratio $x=R / r^{*} . m$ is shown as a parameter.

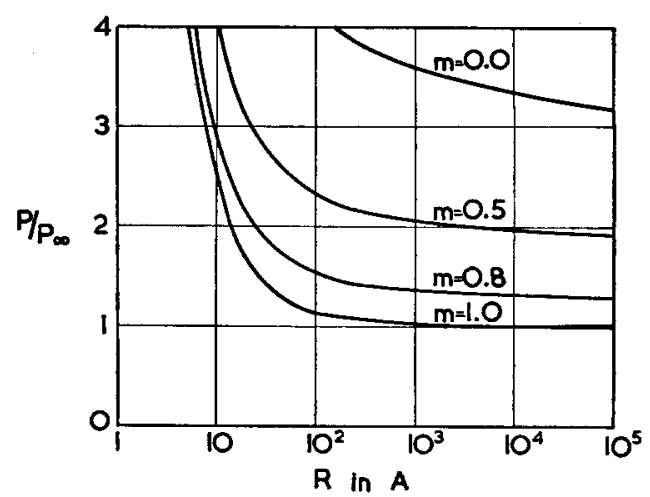

FIg. 3. Supersaturation $P / P_{\infty}$ at which condensation occurs on a spherical particle of radius $R$ at temperature $0^{\circ} \mathrm{C}$. Parameter is $m=\cos \theta$.

If we suppose the nucleating particle to have a radius $R \mathrm{~cm}$, then the nucleation rate per particle is

$$
J=4 \pi 10^{25} R^{2} \exp \left(-\Delta G^{*} / k T\right) .
$$

Now we shall be interested not in the rate itself, but in the temperature (or supersaturation) at which this rate becomes appreciable. We shall define an appreciable rate as one embryo per particle per second $\left(J=1 \mathrm{sec}^{-1}\right)$, recognizing that a few orders of magnitude change in the definition will have negligible effect on the result. Combining this definition with Eqs. (10) and (13), we find for the critical motive free energy $\Delta G_{v}$ at which nucleation becomes appreciable,

$\Delta G_{v}{ }^{2}=[(8 \pi) / 3] \sigma_{12}{ }^{3} f(m, x) / k T\left[60.1+4.604 \log _{10} R\right] .(14)$

Once $\Delta G_{v}$ is expressed in terms of a supercooling or a supersaturation, Eq. (14) gives the complete solution to our problem. The result is quite general and applies to spherical nuclei in almost any situation, the only modification required for unusual cases being in the numerical factors in the square brackets (i.e., in the logarithm of the kinetic coefficient $4 \pi K R^{2}$ ).

In the following sections we shall apply Eq. (14) to a discussion of the condensation, sublimation, and freezing of water as typical examples.

\section{CONDENSATION}

In the condensation process the transition is from supersaturated vapor to liquid. If we denote by $P$ the partial pressure of water vapor in the surrounding gas and by $P_{\infty}$ the saturated vapor pressure over a plane water surface at the same temperature, then assuming the liquid to be incompressible,

$$
\Delta G_{v}=\left(-k T / V_{L}\right) \log \left(P / P_{\infty}\right),
$$

where $V_{L}$ is the volume occupied by a water molecule in the bulk liquid. If we consider a temperature of $0^{\circ} \mathrm{C}$ for convenience, then $\sigma_{12}=75.6 \mathrm{erg} \mathrm{cm}^{-2}$ and (14) becomes

$$
\left(\log \frac{P}{P_{\infty}}\right)^{2}=\frac{1.005 f(m, x)}{1+0.0767 \log _{10} R}
$$


This equation is easily solved by successive approximations. For given values of $m$ and $x$ and an arbitrary $R, P / P_{\infty}$ is calculated. From this a better approximation to $R$ is found from

$$
R(P)=x r^{*}(P)=2 x \sigma_{12} V_{L} / k T \log \left(P / P_{\infty}\right),
$$

and this $R$ in (16) gives a better value of $P / P_{\mathrm{co}}$. This process converges very rapidly and two successive approximations are usually sufficient.

In Fig. 3 we have plotted the results of these calculations over a wide range of conditions. When $m=1.0$ the nucleus is completely wet by water and behaves as a droplet of water of radius $R$. For lower values of $m$ considerable supersaturation is required for condensation even on quite large particles, and these are unlikely to be of any importance in condensation phenomena in the atmosphere. At saturation ratios above 4.2 , nucleation occurs on ions, so that any nuclei requiring more than this supersaturation cannot be activated. For most nuclei the size effect becomes marked for radii less than about $200 \mathrm{~A}$, though this demarkation depends upon the scale considered.

\section{SUBLIMATION}

Sublimation is a very important process in the production of rain from supercooled clouds. If sublimation nuclei become active in the cloud they form ice crystals which grow at the expense of the surrounding water droplets, eventually falling as snow, or melting to give rain. In this case the atmosphere is very close to water saturation, and because of the supercooling, is supersaturated with respect to ice. The motive free energy is then

$$
\Delta G_{v}=-\left(k T / V_{s}\right) \log \left(P_{L} / P_{s}\right)
$$

where $V_{S}$ is the volume of an ice molecule, $P_{L}$ is the vapor pressure over a flat water surface, and $P_{s}$ that over a flat ice surface at the temperature considered.

Equation (18) is not, however, the most general form of the motive free energy for a solid embryo, for there exists the possibility of elastic strain to bring the embryo lattice into partial conformity with the substrate lattice. Turnbull and Vonnegut ${ }^{5}$ have considered this aspect in general terms and have discussed two special cases. If the two lattices are almost identical in the surface plane, then nucleation may be coherent, the embryo forming pseudomorphically with consequent internal strain. This strain energy contributes a term $c \delta^{2}$ to $\Delta G_{v}$, where $\delta$ is the misfit between the two lattices. On the other hand, if the lattices are dissimilar or if the misfit is large, nucleation will not be coherent. In this case dislocations are formed at the junction of the two lattices, increasing the free energy $\sigma_{23}$ of that surface, but there is no contribution to $\Delta G_{v}$.

In practice it is probable that a combination of these two effects occurs, and in addition it is likely that the strain is inhomogeneous, giving a more complex term

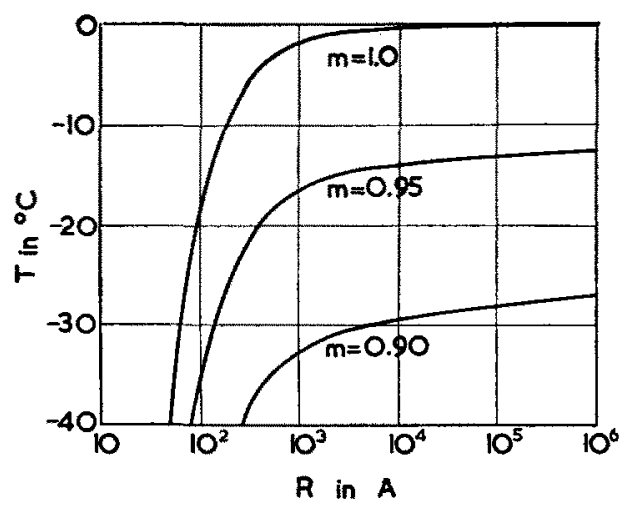

FIg. 4. Temperature $T$ at which sublimation occurs on a spherical particle of radius $R$ at water saturation. Parameter is $m=\cos \theta$.

than $c \delta^{2}$. While either of the two simple cases can be treated easily on the present theory, we shall confine our calculations to the noncoherent case in which $\Delta G_{v}$ is given by (18). The effect of the dislocation content of the 2-3 boundary is automatically taken into account through the value of $m$.

If we denote by $\Delta T$ the supercooling $273^{\circ}-T$ then it is a good approximation to write

$$
\log \left(P_{L} / P_{S}\right)=\alpha \Delta T,
$$

where $\alpha$ has the value $0.00972 /{ }^{\circ} \mathrm{C}$. Equation (14) can then be written,

$$
\Delta T^{2}=\frac{8 \pi \sigma_{12}{ }^{3} V_{s}^{2} f(m, x)}{3(k T)^{3} \alpha^{2}\left(60.1+4.604 \log _{10} R\right)} .
$$

Again this equation can be solved by successive approximations, which must now be carried out both for $R$ and $T$. The resulting curves are shown in Fig. 4, assuming a value of $100 \mathrm{erg} \mathrm{cm} \mathrm{cm}^{-2}$ for $\sigma_{12}$. It is seen that the contact angle of ice on the nucleus must be fairly small (say less than $30^{\circ}$ ) if the substance is to act as a nucleus at a reasonable supercooling.

The curve for $m=1.0$, i.e., for a nucleus completely "wet" by ice, agrees almost exactly with that calculated by Reiss $^{8}$ for this case on a kinetic basis. Curves for other values of $m$ are very similar in shape but depressed in temperature. It can be seen that here the size effect becomes noticeable for particle radii less than about $1000 \mathrm{~A}$. This is a point of some importance since many artificially produced smokes have particle sizes of this order, and size effects may contribute appreciably to their behavior.

Though we shall not show calculations for the case when nucleation is assumed to be coherent, we can make some general observations about the nature of the curves. In the first place the curve $m=1.0$ does not approach $T=0^{\circ} \mathrm{C}$ for large $R$, but instead approaches a lower temperature which is determined by the degree of misfit and the elastic constants of ice.

\footnotetext{
${ }^{8}$ H. Reiss, J. Chem. Phys. 18, 529 (1950).
} 


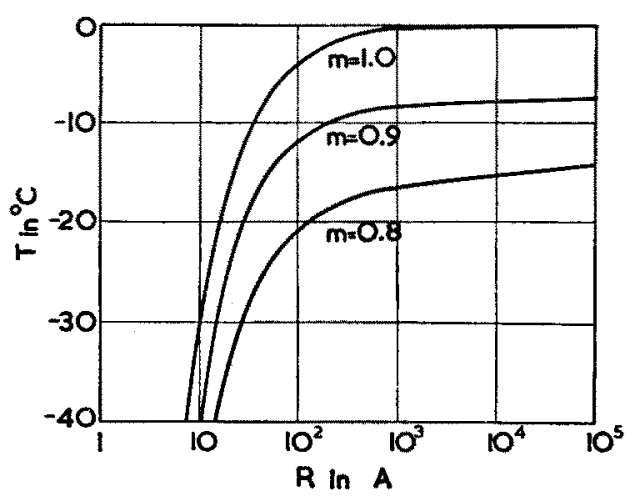

Fig. 5. Temperature $T$ at which freezing occurs on a spherical prarticle of radius $R$ suspended in water. Parameter is $m=\cos \theta$.

The shape of the curve is little altered, and to a first approximation it is merely shifted downwards through this temperature difference. Curves for other values of $m$ are similarly shifted downwards by approximately the same amount. Turnbull and Vonnegut ${ }^{5}$ calculate this shift to be about $-1.5^{\circ} \mathrm{C}$ for $1 \%$ misfit on the basal plane, and of course the shift increases as the square of the misfit.

\section{FREEZING}

In the atmosphere, freezing is a two-stage process, in that condensation must first occur, following by freezing. This immediately limits possible freezing nuclei to those that are also good condensation nuclei, since the supersaturations involved are always small. We can, however, consider also a suspension of the particles in water, in which case the initial condensation process is bypassed.

The discussion is now exactly as for the case of sublimation except that $\sigma_{12}$ for an ice-water interface must be used. This involves considerable uncertainty which has been discussed elsewhere. ${ }^{9-11}$ For our present

${ }^{9}$ B. J. Mason, Quart. J. Roy. Meteorol. Soc. 78, 22 (1952).

${ }^{10} \mathrm{~J}$. E. McDonald, J. Meteorol. 10, 416 (1953).

1 B. J. Mason, J. Meteorol. 11, 514 (1954). calculation, we adopt a value of $20 \mathrm{erg} \mathrm{cm}^{-2}$ which gives reasonable agreement with the observed homogeneous nucleation near $-40^{\circ} \mathrm{C}$ and does not differ very much from theoretical estimates. Figure 5 is calculated on this basis. The remarks we made about coherent nucleation in the case of sublimation apply equally to these curves.

\section{CONCLUSION}

An expression has been developed which takes account of surface properties and particle size in heterogeneous nucleation. The nucleation process is very sensitive to changes of interfacial energy and hence to details of the nucleus surface. Nuclei whose surface planes have symmetry and spacing like those of the embryo and whose bonding forces are similar should exhibit low contact angles and good nucleating properties. Any contamination on the surface which changes this contact angle may be expected to have a considerable effect on the nucleating properties.

Curves of nucleation efficiency as a function of particle size have been calculated for condensation, sublimation, and freezing of water vapor and show that size effects are small for particles greater than $1000 \mathrm{~A}$ in radius. These curves have been drawn over wide ranges of the parameters and the assumptions are clearly invalid at the lowest radii shown. No great accuracy is claimed for the curves since some of the interfacial free energies are rather uncertain.

The assumptions of the theory are only those common to nucleation theory and are considered reasonable. The assumption of spherical nuclei is a reasonable approximation to the usual practical case, and even when this is not true the influence of nucleus shape will not be large unless extreme geometries are encountered.

\section{ACKNOWLEDGMENT}

The author wishes to thank Dr. R. G. Wylie of th National Standards Laboratory for several discussions relating to this work. 